CLAWAR 2020: 23rd International Conference on

Climbing and Walking Robots and the Support

Technologies for Mobile Machines,

Moscow, Russian Federation, 24-26 August 2020.

https://doi.org/10.13180/clawar.2020.24-26.08.44

\title{
AGRICULTURAL ROBOTICS: A STATE OF THE ART SURVEY
}

\author{
LUIZ F. P. OLIVEIRA, MANUEL F. SILVA and ANTÓNIO P. MOREIRA \\ Centre for Robotics in Industry and Intelligent Systems (CRIIS), INESC TEC, \\ Rua Dr. Roberto Frias, Porto, 4200-465 Porto, Portugal \\ Department of Electrical Engineering, School of Engineering of the Polytechnic of Porto 6 INESC TEC \\ Rua Dr. António Bernardino de Almeida, 431, Porto, 4249-015 Porto, Portugal \\ Department of Electrical and Computer Engineering, Faculty of Engineering of the University of Porto 8 \\ INESC TEC \\ Rua Dr. Roberto Frias, s/n, Porto, 4200-465 Porto, Portugal \\ E-mail: luizoliveira@ieee.org,mss@isep.ipp.pt,antonio.p.moreira@inesctec.pt \\ http://criis.inesctec.pt/
}

\begin{abstract}
The constant increase in the world population has progressively demanded that humanity develop new technologies to face challenges such as providing high-quality food to the consumer market. In this sense, the concept of precision agriculture arises, proposing the development of agricultural activities such as preparing the land, sowing, planting, treating plants and harvesting automatically through robotic systems. This study focuses on performing a systematic review of the state of the art of robotics applications to execute agricultural activities. Through a comparative analysis of the existing solutions it was possible to highlight the similarities, differences and limitations of several agricultural robots. After looking at the needs of agricultural tasks and the limitations of robots, the challenges that are still unresolved and their possible solutions are indicated.
\end{abstract}

Keywords: Agricultural robots; Agriculture 4.0; Precision agriculture.

\section{Introduction}

There are currently about 7.6 billion people on the planet, and by 2050 this number is expected to increase to 9.8 billion people. ${ }^{1}$ The growth in the world population brings with it several types of issues, such as the need to increase food production in increasingly smaller agricultural environments, since around $68 \%$ of the world population will live in urban environments by $2050^{2}$ and will need twice the current food production capacity. ${ }^{3}$ The percentage of arable land has significantly decreased, since in 1991 it represented around $39.47 \%$ of the land area and in 2013 it reduced to $37.7 \%{ }^{4}$ With the proposal to improve people's living conditions, the increase in the global urbanization process tends to increase individual's financial income, which - consequently - makes people seek a healthier way of life and nutrition. ${ }^{5}$ As a proposal to solve the increasing demand for high quality food, food producers around the world are looking for cost-saving methods to stay in the competitive agricultural industry market. Rural producers are automating agricultural activities to maximize their profits, because about $39 \%$ of the costs of certain North American farms are destined to the payment of the workforce and due to the lack of qualified employees to work on the farms. ${ }^{6}$ Thus, the concept of precision agriculture has increasingly gained prominence with the use of automated or robotic systems for carrying out daily activities in the ground, such as: land preparation, sowing, planting, pest control and harvesting. ${ }^{7}$ Therefore, this study presents a systematic review of the main agricultural activities that have being automated through robotic systems, aiming to expose not only the current technological challenges, but also to suggest possible future solutions. 


\section{Robotic Applications in Agriculture}

Combined with the growth of research in the agricultural field, scientific and technological advances in the areas of mobile robotics, computer vision and artificial intelligence allows the development of increasingly accurate activities. Tasks such as harvesting, pruning and spraying cultivation areas are chosen to be improved precisely because such activities are performed manually, involving labor costs and over-application of expensive chemicals (increasing environmental impacts). On the other hand, the task of estimating food production allows the agricultural producer to have greater control over their production, identifying possible situations such as diseases on the plantation that are impossible to be detected by naked eye. ${ }^{5}$ In this sense, the following subsections will address various types of robots (some shown in the Fig. 1) associated with each agricultural task individually.

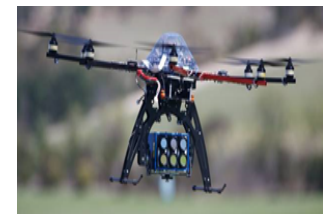

(a) Sowing. ${ }^{8}$

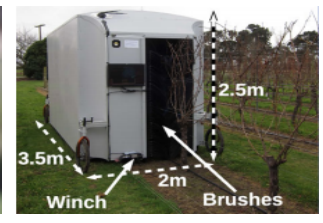

(b) Pruning. ${ }^{9}$

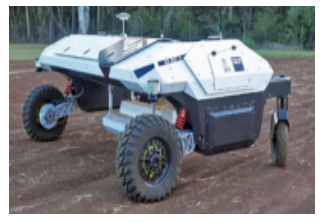

(c) Weeding. 10

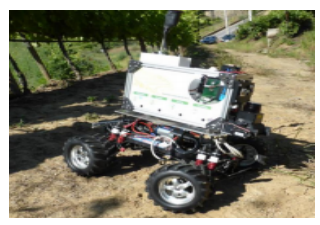

(d) Yield estimation. ${ }^{11}$

Fig. 1. Examples of robotic applications in agriculture.

\subsection{Robotic Applications for Land Preparation before Planting}

Among various functions, the Cäsar robot can perform the task of fertilizing the soil both remotely controlled by a human and autonomously. ${ }^{12}$ To navigate the plantation, it uses Real Time Kinematic (RTK) technology for the Global Navigation Satellite System (GNSS), capable of improving location and positioning accuracy to about $3 \mathrm{~cm}$. With a four-wheel steering system (4WS) to maximize its maneuverability, the Greenbot robot was the first commercial robot completely used to perform repetitive tasks such as sowing, plowing or fertilizing 10 hours a day, transporting up to $750 \mathrm{~kg}$ and $1500 \mathrm{~kg}$ in the front and rear compartments, respectively. ${ }^{13}$ It has a bump sensor to detect obstacles ahead and make emergency stops.

\subsection{Robotic Applications for Sowing and Planting}

To accelerate the sowing process of agricultural fields, a mobile robot powered by continuous tracks (to carry heavy loads on non-uniform soils) was developed to transport a reservoir containing general seeds and to carry out its uniform distribution throughout the plantation. ${ }^{14}$ Another robot of large wheat precision sowing is the 4WS robot, which was able to sow with $93 \%$ precision even for different locomotion speeds. ${ }^{15}$ Unlike Srinivasan et al. (2016), the four-wheel-drive (4WD) robot was designed to be light weighted (to prevent soil deterioration) and to perform corn seeding through an individual seed selector, capable of distributing the quantity of seeds suitable for planting. ${ }^{16}$

\subsection{Robotic Applications for Plant Treatment}

After the planting stage, keeping agricultural products healthy until harvest is not always a simple task, as it is necessary to take care of the plantation so that diseases do not arise and spread throughout the crops as a whole and, consequently, turn the harvest unfeasible. With the purpose of detecting multiple diseases (powdery mildew and tomato spotted wilt virus) 
in greenhouses, the authors Schor et al. (2016) developed a robotic system composed of a 6 degrees-of-freedom (DOF) manipulator on a fixed platform with a Red, Green, Blue (RGB) camera and a laser distance sensor (DT35, SICK). The manipulator is used to move the RGB camera around the concerned plant and the laser sensor is employed to measure the distance from the manipulator to the plant, to avoid collisions. After capturing the images, the disease detection process is initiated through Principal Component Analysis (PCA) and Coefficient of Variation (CV). ${ }^{17}$ As a result, the system obtained a $64 \%$ accuracy rate for the classification of plants with low level of powdery mildew disease and $90 \%$ for tomato spotted wilt virus disease, indicating the possibility of early identification with high precision of disease detection.

Automated seeding robotic systems, guided by the GNSS must consider possible situations of signal loss, such as the technologies of Global Position Systems (GPS) (North American), GLONASS (Russian) or GALILEO (European). ${ }^{18,19}$ In order to keep the health of vineyards constant throughout the cultivation, an Unmanned Aerial Vehicle (UAV) with a GPS data-based navigation system and an Inertial Measurement Unit (IMU) capable of obtaining thermal and multispectral images was used to calculate the indices of wine-growing vegetation, based on the Normalized Difference Vegetation Index (NDVI), to monitor the soil and efficiently manage the plantation irrigation system, ${ }^{8}$ according to Fig. 1(a). The mapping of crop fields can also be performed by UAV, because unlike the monitoring of fields made by satellites and airplanes, UAV have a low flight altitude, capturing high resolution images without the interference of clouds. Thus, through an UAV equipped with a high resolution color camera, researchers Torres-Sánchez et al. (2014) performed multi-temporal mapping of a fraction of vegetation in wheat fields at the beginning of the season, and application of herbicide to lawn. ${ }^{20,21}$ Through various indices (CIVE, ExG, ExGR, Woebbecke Index, NGRDI, VEG) they studied the behavior of vegetation before and after sowing. The technology proposes to assist planting monitoring and weed identification activities.

An additional way to prevent the proliferation of diseases and/or weeds before harvesting is through the use of pesticides/herbicides. Compared to a speed or wide area sprayer, UAV can reduce the use of pesticides and maximize the efficiency of cultivation areas. ${ }^{5,22}$ A quadcopter, located just a few meters from the plantation, capable of carrying up to 5 liters of Urea (organic compound), was used to spray such product over a defined region. ${ }^{23}$ Through human-machine interaction, a robot was developed to remotely spray pesticides (which pose risks to human health) on vineyards, using a Human Machine Interface (HMI) ${ }^{24}$ In order to control the spraying within certain areas, the authors Berenstein and Edan (2018) developed a system for opening a valve connected to a mobile wheeled robot, which moved through a vineyard. ${ }^{25}$ Using an RGB camera and distance sensors, the robot calculates the opening diameter of the valve that releases the pesticide, based on the machine vision Foliage Detection Algorithm (FDA) and Grape clusters Detection Algorithms (GDA), resulting in a $45 \%$ material reduction. For the types of crops that depend on pollination activities - such as kiwi cultivation - researchers have developed a mobile wheeled robot, with a system divided into: machine vision (implementing Convolutional Neural Network $(\mathrm{CNN})$ ), flower tracking (which has a strong dependence on the odometric system), spray time control and spray dispenser (composed of 20 nozzles). ${ }^{26}$ For this task, the robot was able to pollinate about $79.5 \%$ of the kiwi flowers at a speed of $3.5 \mathrm{~km} / \mathrm{h}$. The task of pruning grapevines was also studied by researchers, so this process could be achieved through a robotic system. ${ }^{9}$ In this case, a mobile platform containing wheels was employed (dragged by a cable attached to a tractor, as shown in Fig. 1(b)) that surrounds the vineyard, which has a 6-DOF manipulator, Light-Emitting Diode (LED) lighting and three RGB cameras, all fixed to the platform. In order to prune the vines, the 6-DOF manipulator contains a cutter as the final actuator. In addition to the mobile platform, several computer vision 
techniques, such as foreground/background segmentation, detection of 2D structures, correspondence and incremental 3D reconstruction were applied. The Support Vector Machine (SVM) learning algorithm was also used to classify the different types of detectable objects, that is, branches or poles and fixing wires. In order to prune specific branches, the system implements a collision-free path planner, based on Rapidly Exploring Random Tree (RRT), RRT-Connect. As a result, the researchers reported that through real application testing, the system was able to cut the branches of the vines. ${ }^{9}$

With the increase in the demand for organic products (without the use of herbicides), producers are looking for new ways to carry out weed control in plantation crops. The researchers Gai et al. (2020) proposed such control to be done through a robotic system, capable of detecting weeds near - or even within - broccoli and lettuce plantations. Plantation data were extracted using the Kinect v2 sensor. ${ }^{27}$ The study focused on the activities of vegetation pixels segmentation (using the Random Sample Consensus (RANSAC)), plant extraction (2D connected-component method), resource extraction (length, width and height of leaves, arrangement of ribs and area) and finally the classification of plants (based on characteristics). Through a remotely controlled vehicle, the real tests presented a high detection rate of broccoli $(91.7 \%)$ and lettuce $(90.8 \%)$, however the authors did not describe the stage of weed removal. Unlike Gai et al., the researchers McCool et al. (2018) developed the AgBot II robot, as shown in Fig. 1(c), with the task of removing weeds from crops using three mechanical tools: an arrow-shaped hoe, a toothed tool and a cutting tool, all based on the implementation of the Local Binary Pattern (LBP) and Covariance Feature techniques in images collected by a color camera. ${ }^{10}$ To take advantage of the constant exposure to the sun while carrying out weeding activities, the RIPPA ${ }^{28}$ and Ladybird ${ }^{29}$ robots were designed to contain photovoltaic panels in their mechanical structure and, thus, recharge their battery systems. The BoniRob robot performs both tasks (classification between plantation and weeds and their subsequent removal, using sprays and a ramming rod) by merging the images collected through camera and ultrasonic sensors. ${ }^{30}$

\subsection{Robotic Applications for Harvesting}

In order to evaluate the various robotic systems for harvesting fruits, Bac et al. (2014) elaborated several performance indicators, such as harvest success, success in capturing ripe fruit, damage rate and time of operation. After analyzing about 50 projects, they concluded that most of the applications involving robots in harvesting activities used: field tested mobile robots, manipulators with 3-DOF, RGB and/or multi-spectral cameras and adaptive algorithms (to suit the changes in objects, environment and/or market requirements). ${ }^{31}$

Committed to the strawberry harvesting, Ge et al. (2019) developed the location of fruits and perception of the environment by means of a robot equipped with an RGB-Depth (RGB-D) camera. The researchers focused on the development of three tasks: the detection of strawberries through deep learning networks (Region-based Convolutional Neural Network (R-CNN)); a trajectory planning algorithm to plan the collision-free action of picking strawberries based on $2 \mathrm{D}$ images and the $3 \mathrm{D}$ point cloud; tests performed in a real environment. After carrying out several experiments, among all the ripe strawberries identified, $74.1 \%$ were successfully harvested with an F1-score (harmonic mean of accuracy and recall) of 0.9. ${ }^{32}$ Through the Vegebot robot, English researchers developed a lettuce collection mechanism. ${ }^{33}$ There are three types of challenges for this kind of task: removal, protection and identification of lettuce heads. In this case, the researchers developed a closed-loop control method to monitor the force required to extract the lettuce. Since lettuce is a very sensitive vegetable, two RGB cameras located above and at $45^{\circ}$ of the vegetation were used to identify and prevent damage to the vegetable. R-CNN was also used - due to its high 
detection rate - to allow its later use in systems with low financial cost and processing power. ${ }^{33}$ After identifying the center (head) of the lettuce, a manipulator system with 6DOF positions the lettuce device extractor over the vegetable and removes it. The system located (with $91 \%$ success rate) and classified (with a $82 \%$ accuracy) vegetables when tested with a large amount of tests. ${ }^{33}$

\subsection{Robotic Applications for Yield Estimation}

Without the need to harvest the fruits, the robots used to estimate crop yields focus their efforts on improving computer vision systems. A test platform called Shrimp equipped with six RGB cameras was used as a ground wheeled vehicle to detect and estimate the yield of apple orchards. ${ }^{34}$ Unlike Botterill et al. (2017) - who used a platform to completely cover the vineyard - Bargoti and Underwood (2017) performed the monitoring of apples exposed to natural lighting conditions, considered a challenging task. ${ }^{34}$ The Shrimp vehicle has a GPS system and an Inertial Navigation System (INS), used to estimate the robot's position and locate each sampled image. As proposed by Bac et al. (2014), Bargoti and Underwood (2017) implemented two distinct learning algorithms - Multiscale Multilayer Perceptron (MLP) and CNN. ${ }^{34}$ They also implemented two different techniques for the detection of apples, the Watershed (WS) segmentation algorithm and the Circular Hough Transform (CHT). The best results were achieved using CNN and WS, reaching an apple detection of $82.5 \%$, with an F1-score of 0.791 and a coefficient of determination $r^{2}$ (the goodness of fit for a given model) of $0.826 .{ }^{34}$

Another work of great relevance was the development of a wheeled mobile robot to monitor the cultivation of grapes in the Douro region in Portugal, which presents steep slope vineyards. ${ }^{11}$ Equipped with RGB cameras and infrared (IR) sensors, Light Detection And Ranging (LiDAR) and encoders, the 4WD robot - called Agrob V14 - as shown in Fig. 1(d), was designed to work autonomously even in the event of GNSS signal unavailability, since the characteristics found in the Douro vineyards reduce dramatically the availability and accuracy of the GNSS service. ${ }^{11}$ The characteristics of the region's soil, with a high content of stone elements, also impose restrictions on the planning of trajectories (strongly dependent on the information provided by odometer and IMU systems). It proposes a new approach to the Simultaneous Localization And Mapping (SLAM) technique by inserting Radio-Frequency IDentification (RFID) tags located at the beginning and end of each row of the vineyard to allow the reduction of the complexity of the 2D Extended Kalman Filter (EKF) used, and increase computational efficiency, this process being named VineSLAM. The authors conclude that the Agrob V14 robot can overcome ditches, rocks and high slopes $(30 \%)$, although these characteristics impose difficulties to the robot, which reduces the detection of natural characteristics by the Laser Range Finder (LRF). ${ }^{35}$

\section{Discussion of Existing Applications}

The progressive approach of the main scientific works existing in the area of precision agriculture, analyzing applications of robotics from the preparation of the land before planting, sowing and planting, and the treatment of plants until the harvest stage, was elaborated so that it was possible to identify the advances, trends and limitations existing to date, and to establish possible unresolved issues.

\subsection{Comparison between Existing or On-going Solutions}

In order to summarize all the applications described in the present study, and to visualize their similarities and differences, Table 1 lists the task performed by each robot previously described. 
Table 1. Comparison between existing or on-going solutions.

\begin{tabular}{|c|c|c|c|c|c|}
\hline Application & Robot & $\begin{array}{l}\text { Locomotion } \\
\text { System }\end{array}$ & $\begin{array}{c}\text { Final } \\
\text { Application }\end{array}$ & Used Sensors & $\begin{array}{l}\text { Computer } \\
\text { Vision } \\
\text { Algorithms }\end{array}$ \\
\hline \multirow{2}{*}{$\begin{array}{c}\text { Land } \\
\text { Preparation }\end{array}$} & Cäsar ${ }^{12}$ & $4 \mathrm{WD}$ & Orchard or Vineyard & RTK GNSS & Not included \\
\hline & Greenbot $^{13}$ & $4 \mathrm{WS}$ & $\begin{array}{l}\text { Horticulture, Fruit and } \\
\text { Arable Farming }\end{array}$ & $\begin{array}{c}\text { RTK/GPS, } \\
\text { Bump Sensor }\end{array}$ & Not included \\
\hline \multirow{3}{*}{ Sowing } & Land Robot ${ }^{14}$ & Caterpillar Treads & $\begin{array}{l}\text { Seeds in } \\
\text { General }\end{array}$ & Ultrasonic Sensor & Not included \\
\hline & Lumai-5 $5^{15}$ & $4 \mathrm{WS}$ & Wheat & Speed, Pressure and IR & Not included \\
\hline & Land Robot ${ }^{16}$ & $4 \mathrm{WD}$ & Corn & Ultrasonic Sensor & Not included \\
\hline \multirow{10}{*}{ Treatment } & Fixed Robot ${ }^{17}$ & Manipulator & Bell Pepper & RGB Camera and Laser & $\mathrm{PCA}$ and $\mathrm{CV}$ \\
\hline & Octocopter $^{8}$ & UAV & Grape & Multispectral Camera & NDVI \\
\hline & Quadcopter $^{20}$ & UAV & General Farms & RGB Camera & Otsu Method \\
\hline & AgriRobot and SAVSAR ${ }^{24}$ & $4 \mathrm{WD}$ & Grape & $\begin{array}{l}\text { RGB Camera } \\
\text { and LiDAR }\end{array}$ & FDA and GDA \\
\hline & Spray Robot ${ }^{25}$ & $4 \mathrm{WD}$ & Grape & $\begin{array}{l}\text { RGB Camera } \\
\text { and Laser }\end{array}$ & FDA and GDA \\
\hline & Pollinator Robot ${ }^{26}$ & $4 \mathrm{WD}$ & Kiwi & RGB Camera and Odometry & $\mathrm{CNN}$ \\
\hline & Land Robot ${ }^{27}$ & $4 \mathrm{WD}$ & Weed & Kinect v2 & RANSAC \\
\hline & AgBot $\mathrm{II}^{10}$ & $4 \mathrm{WS}$ & Weed & RGB Camera & LBP \\
\hline & BoniRob $^{30}$ & $4 \mathrm{WS}$ & Weed & $\begin{array}{l}\text { RGB Camera and } \\
\text { Ultrasonic Sensor }\end{array}$ & $\mathrm{CNN}$ \\
\hline & RIPPA $^{28}$ and Ladybird ${ }^{29}$ & $4 \mathrm{WS}$ & Weed & $\begin{array}{c}\text { Hyperspectral and Thermal } \\
\text { Cameras, RTK/GPS/INS } \\
\text { and LiDAR }\end{array}$ & ExG-ExR \\
\hline \multirow{2}{*}{ Harvest } & Noronn $\mathrm{AS}^{32}$ & $4 \mathrm{WS}$ & Strawberry & RGB-D Camera & R-CNN \\
\hline & Vegebot $^{33}$ & $4 \mathrm{WD}$ & Lettuce & RGB Camera & $\mathrm{R}-\mathrm{CNN}$ \\
\hline \multirow{2}{*}{$\begin{array}{c}\text { Yield } \\
\text { Estimation }\end{array}$} & Shrimp ${ }^{34}$ & Mobile Platform & Apple & RGB Camera & $\overline{\mathrm{MLP} \text { and } \mathrm{CNN}}$ \\
\hline & Agrob V14 11,35 & $4 \mathrm{WD}$ & Grape & RGB Camera and LiDAR & SVM \\
\hline
\end{tabular}

By analyzing Table 1, it is possible to observe that most robots present a $4 \mathrm{WD}$ system. This is due to its ease of construction, control and the vast majority of the plantation soils that are not as rough and/or steep. It is noted that unlike the sowing robots, all robots associated with the treatment of the plantation have some computer vision algorithm, showing the different levels of difficulty between each agricultural activity. Due to the difficulty of developing an accurate and reliable system that replaces manual labor, most of the reviewed studies sought to build robots with a low-cost computer vision system, that is, using conventional RGB cameras. However, the studies still remain in the research area, with no commercial use on a large scale, except the Cäsar and Greenbot robots. It is also observed that in most applications that have a camera vision system, the CNN method is used as a way of classifying objects and/or fruits, due to the intense technological advances in the area of computing, increasing the performance of computer systems and allowing them to perform mathematical calculations more quickly. Unlike an urban environment, the agricultural environment requires, in addition to the aforementioned tasks, a greater robust electronics/mechanics of the robots, allowing them to continue operating normally even under variations in temperature and humidity, dust incidence, vibration (uneven soil) and dry or wet soil (after planting irrigation). Even with the various existing robotic systems, it is possible to observe its limitations and stipulate new directions for the development and improvement of new robots applied to the concept of precision agriculture.

\subsection{Unsolved issues}

In general, it is possible to group open challenges and/or issues into three categories related to the robot, navigation systems and computer vision and system intelligence.

As noted in Table 1, most of the employed robots use a 4WD system. However, robotic systems with wheels are strongly affected by terrains containing stone elements and/or cavities, because for their locomotion the wheels need to be in constant contact with the soil. For this reason, the constant locomotion of such robots throughout the plantation 
results in a high rate of soil deterioration. ${ }^{16}$ In this sense, the improvement of the energy consumption of UAV and, therefore, its flight time, ${ }^{22}$ enabled its greater efficiency in carrying out agricultural tasks without contact with the soil. Another research possibility is the use of robots with other forms of locomotion, ${ }^{31}$ namely legged robots, as they are able to move in irregular and unstable terrain. ${ }^{36,37}$ In addition, they do not need constant contact with the ground for movement, and the damage caused to the plantation soil is much less than that of wheeled robots. Since only Cäsar and Greenbot robots are commercially available, another proposal would be the development of a new research based on legged robots already available for use, that is, off-the-shelf. As examples of off-the-shelf robots, two stand out: $\mathrm{ANYmal}^{38}$ and SPOT. ${ }^{39}$ Both these robots are quadrupedal and have their own solutions for navigation systems, trajectory planning, computer vision and have an intuitive programming interface for the development of new applications by researchers.

Regarding the development of robots with navigation systems based on GNSS signals in agricultural plantations whose height of vegetation or the conditions of the local environment impair the reception of GNSS signals, such as wine-growing activities in the Douro region in Portugal, the improvement of SLAM techniques ${ }^{11}$ may contribute to a more efficient automated locomotion, estimating with greater precision the location and position of the robot in relation to the environment surrounding it.

Finally, as we are going through the process of the 4th industrial revolution, applications in cyber-physical systems have been in great demand by large companies in the sector. In this sense, the integration between the various applications of agricultural robots and the Internet of Things (IoT) devices/sensors, ${ }^{5}$ can together maximize the control and monitoring of crops, sharing machine to machine (M2M) information, and displaying them in a simple form to the rural producer.

\section{Conclusions}

Through a systematic review of various applications of robots in the agricultural field, was carried out a gradual approach to the theme of robots performing tasks of preparing the land, sowing, planting, plant treatment and harvesting. Through a discussion of why such systems or techniques are more appropriate to perform certain tasks, for precision agriculture closer to real field conditions, improvements in locomotion, navigation and vision systems in rough terrains are required, in addition to greater integration between the various robotic systems and IoT technologies. Therefore, the present study sought to contribute to the state of the art of agricultural robot applications, transforming large farms into smart farms.

\section{Acknowledgments}

This work is financed by National Funds through the Portuguese funding agency, FCT Fundação para a Ciência e a Tecnologia, within project UIDB/50014/2020.

\section{References}

1. U. Nations, World population projected to reach 9.8 billion in 2050 (2020), https://www . un. org/development/desa/en/news/population/world-population-prospects-2017.html.

2. U. Nations, Economic \& Social Affairs 1, 1 (2018).

3. X. Zhang and E. A. Davidson, Improving nitrogen and water management in crop production on a national scale, in AGU Fall Meeting Abstracts, dec 2018.

4. L. Zhang, I. K. Dabipi and W. L. Brown Jr., Internet of Things Applications for Agriculture (John Wiley \& Sons, Ltd, 2018).

5. M. Ayaz, M. Ammad-Uddin, Z. Sharif, A. Mansour and E. M. Aggoune, IEEE Access 1 (2019).

6. C. F. B. Federation, Still searching for solutions: Adapting to farm worker scarcity survey 2019 (2020), https://www.cfbf.com/wp-content/uploads/2019/06/LaborScarcity.pdf. 
7. S. A. K. N. Tarannum, M. K. Rhaman and S. R. Shakil, Journal of Modern Science and Technology 3, 88 (2015).

8. D. Turner, A. Lucieer and C. Watson, Development of an unmanned aerial vehicle (uav) for hyper-resolution vineyard mapping based on visible, multispectral and thermal imagery Proceedings of 34th International Symposium on Remote Sensing of Environment 1012011.

9. T. Botterill, S. Paulin, R. Green, S. Williams, J. Lin, V. Saxton, S. Mills, X. Chen and S. Corbett-Davies, Journal of Field Robotics 34, 1100 (2017).

10. C. McCool, J. Beattie, J. Firn, C. Lehnert, J. Kulk, O. Bawden, R. Russell and T. Perez, IEEE Robotics and Automation Letters 3, 1184 (2018).

11. F. N. dos Santos, H. Sobreira, D. Campos, R. Morais, A. Paulo Moreira and O. Contente, Journal of Intelligent \& Robotic Systems 83, 429 (2016).

12. Raussendorf, Fruit robot (2020), https://www.raussendorf.de/en/fruit-robot.html.

13. P. Makers, Greenbot (2020), https://www.precisionmakers.com/en/greenbot-2/.

14. N. Srinivasan, P. Prabhu, S. S. Smruthi, N. V. Sivaraman, S. J. Gladwin, R. Rajavel and A. R. Natarajan, Design of an autonomous seed planting robot, in 2016 IEEE Region 10 Humanitarian Technology Conference (R10-HTC), 2016.

15. L. Haibo, S. Dong, L. Zunmin and Y. Chuijie, Journal of Robotics 1, 1(11 2015).

16. M. U. Hassan, M. Ullah and J. Iqbal, Towards autonomy in agriculture: Design and prototyping of a robotic vehicle with seed selector, in 2016 2nd International Conference on Robotics and Artificial Intelligence (ICRAI), 2016.

17. N. Schor, A. Bechar, T. Ignat, A. Dombrovsky, Y. Elad and S. Berman, IEEE Robotics and Automation Letters 1, 354 (2016).

18. B. Siciliano and O. Khatib, Springer Handbook of Robotics, 2nd edn. (Springer Publishing Company, Incorporated, 2016).

19. N. Khan, G. Medlock, S. Graves and S. Anwar, Gps guided autonomous navigation of a small agricultural robot with automated fertilizing system, in SAE Technical Paper, (SAE International, 04 2018).

20. J. T. Sánchez, J. M. Peña, A. I. Castro and F. L. Granados, Computers and Electronics in Agriculture 103, 104 (2014).

21. H. Xiang and L. Tian, Biosystems Engineering 108, 174 (2011).

22. J. Kim, S. Kim, C. Ju and H. I. Son, IEEE Access 7, 105100 (2019).

23. S. Meivel, K. Dinakaran, N. Gandhiraj and M. Srinivasan, Remote sensing for urea spraying agricultural (uav) system, in 2016 3rd International Conference on Advanced Computing and Communication Systems (ICACCS), 2016.

24. G. Adamides, C. Katsanos, I. Constantinou, G. Christou, M. Xenos, T. Hadzilacos and Y. Edan, Journal of Field Robotics 34, 1407 (2017).

25. R. Berenstein and Y. Edan, IEEE Transactions on Automation Science and Engineering 15, 641 (2018).

26. H. Williams, M. Nejati, S. Hussein, N. Penhall, J. Y. Lim, M. H. Jones, J. Bell, H. S. Ahn, S. Bradley, P. Schaare, P. Martinsen, M. Alomar, P. Patel, M. Seabright, M. Duke, A. Scarfe and B. MacDonald, Journal of Field Robotics 37, 246 (2020).

27. J. Gai, L. Tang and B. L. Steward, Journal of Field Robotics 37, 35 (2020).

28. R. Bogue, Industrial Robot: An International Journal 43, 450(8 2016).

29. J. P. Underwood, M. Calleija, Z. Taylor, C. Hung, J. M. G. Nieto, R. Fitch and S. Sukkarieh, Real-time target detection and steerable spray for vegetable crops2015.

30. X. Wu, S. Aravecchia, P. Lottes, C. Stachniss and C. Pradalier, Journal of Field Robotics 37, $322(2020)$.

31. C. W. Bac, E. J. van Henten, J. Hemming and Y. Edan, Journal of Field Robotics 31 (2014).

32. Y. Ge, Y. Xiong, G. L. Tenorio and P. J. From, IEEE Access 7, 147642 (2019).

33. S. Birrell, J. Hughes, J. Y. Cai and F. Iida, Journal of Field Robotics 37, 225 (2020).

34. S. Bargoti and J. P. Underwood, Journal of Field Robotics 34, 1039 (2017).

35. F. B. N. d. Santos, H. M. P. Sobreira, D. F. B. Campos, R. M. P. M. d. Santos, A. P. G. M. Moreira and O. M. S. Contente, Towards a reliable monitoring robot for mountain vineyards, in 2015 IEEE International Conference on Autonomous Robot Systems and Competitions, 2015.

36. L. F. P. Oliveira and F. L. Rossini, IEEE Latin America Transactions 16, 375(Feb 2018).

37. M. F. Silva and J. T. Machado, Journal of Vibration and Control 18, 1753 (2012).

38. P. Fankhauser, Anymal c (2020), https://www.anybotics.com/anymal-legged-robot/.

39. E. Guizzo, Boston dynamics' spot robot dog goes on sale (2020), https://spectrum.ieee. org/automaton/robotics/industrial-robots. 